Case Report

\title{
Two cases of spindle cell carcinoma with a histopathological review of biphasic tumours of head and neck
}

\author{
Mirza N. ${ }^{1}$, Haider N. ${ }^{2}$, Fatima S. $^{3}$ \\ ${ }^{1}$ Dr. Nihal Mirza, Pathology, Aseer Central Hospital, Abha, KSA, ${ }^{2}$ Dr. Nazima Haider, Pathology, King Khalid \\ University, Abha, KSA, ${ }^{3}$ Dr. Sohaila Fatima, King Khalid University, Abha, KSA.
}

Corresponding Author: Dr. Nazima Haider, Pathology, King Khalid University, Abha, KSA, Email: nazima_haider@yahoo.com

\begin{abstract}
Spindle cell carcinoma (SPCC) is a very rare and unique biphasic variant of squamous cell carcinoma (SCC) which is a very rare malignancy of head and neck region.Spindle cell tumours are challenging feature of head and neck pathology because of different types of spindle cell lesions occurring which can be reactive to benign to malignant and very aggressive. We present here two rare cases of SPCC one involving the nasal cavity in a 38 year female and other a very aggressive maxillary sinus tumour in a 60 year male and discuss its differential diagnosis with an emphasis on biphasic tumours of head and neck.
\end{abstract}

Key words: Spindle cell carcinoma, Head and neck, Biphasic tumours

\section{Introduction}

Spindle cell carcinoma (SPCC) is a very rare and unique biphasic variant of squamous cell carcinoma (SCC) which can be seen to involve any part of the body including head and neck region. It has an epithelial origin but with a mesenchymal appearance hence called spindle cell carcinoma or carcinosarcoma. Nasal cavity and sinuses are very rare sites of involvement $[1,2]$. Spindle cell tumours are challenging feature of head and neck pathology because of different types of spindle cell lesions occurring which can be reactive to malignant and very aggressive. This makes accurate diagnosis difficult.

We presenthere tworare cases of SPCC one involving the nasal cavity in a 38-year female and other avery aggressive maxillary sinus tumour in a 60-year male and discuss its differential diagnosis with an emphasis on biphasic tumours of head and neck.

\section{Case Reports}

Case-1: A 38 year old female presented with history of intermittent epistaxis from left nasal cavity from one year with gradually increasing nasal obstruction from 6 months. There was no history of smoking or alcohol addiction. On examination, the patient had an ulcerated, polypoidal, irregular mass obstructing the complete left nasal vestibule. There was no lymphadenopathy. The patient was weak and anaemic (Haemoglobin- $9 \mathrm{gm} / \mathrm{dl}$ ) Computerized tomography (CT) scan of the head and neck showed a heterogeneously enhancing mass of the left nasal cavity. Biopsy of the mass revealed a malignant spindle cell proliferation (Figure 1) with tumour cells showing hyperchromatic nuclei and prominent nucleoli and eosinophilic cytoplasm. (Figure 2) Immunohistochemistry showed tumour cell to be pan-cytokeratin (CK), vimentin positive. (Figure 3) Smooth muscle actin (SMA), desmin, HMB 45, S100, calponin, CD 34, myogenin were negative. A diagnosis of Spindle Cell Carcinoma (SPCC) of nasal cavity was made. The patient was referred to higher centre for surgical resection and management where tumour was removed by wide local excision. The patient did well post surgery and received chemo radiotherapy. She is currently under follow up from 1 year.

Case-2: A 60-year male was suffering from intermittent epistaxis from 6 months. Then he started to complain of left nasal obstruction from 4 months and slowly developed diplopia. He also complained of anorexia, dysphagia and weight loss in past 1 month. Patient had the addiction of using snuff and smoking. On examination, a diffuse swelling was seen 


\section{Case Report}

below the left eye and cheek measuring $5 \times 4 \mathrm{~cm}$ extending to the hard palate with slight proptosis of left eye. Patient was very weak and anaemic (Hemoglobin $8 \mathrm{gm} / \mathrm{dl}$ ). On rhinoscopy, patient had a large pinkish fleshy mass in left nasal cavity. There was no lymphadenopathy. CT scan and MRI of head and neck showed a large heterogenous mass in the maxillary sinus and with destruction of all its walls and extension to the left orbital cavity with involvement of the medial and inferior rectus muscles. The mass hadalso extended into the nasal cavityand superiorly to the cribriform plate, posteriorly to the left pterygopalatine fossa and the infra-temporal fossa.

There was destruction of the inferior wall of the sphenoid sinus and inferiorly, the mass had destroyed the left side hard palate, extending into the oral cavity (Figure 4). Biopsy of the nasal mass showed malignant spindle cell proliferation in a hemorrhagic background with spindle cells showing hyperchromatic nuclei and prominent nucleoli and eosinophilic cytoplasm. Abundant blood vessels with plump endothelial cells and neutrophils were seen mimicking granulation tissue (Figure 5).

Immunohistochemistry showed tumour cell to be pan-cytokeratin (CK), vimentin, smooth muscle actin (SMA) positive. However desmin, HMB 45, S100, calponin, CD31, CD 34, myogenin, CD 99 were negative. A diagnosis of SPCC of maxillary sinus was made.

Squamous cell component was not seen in both the cases.

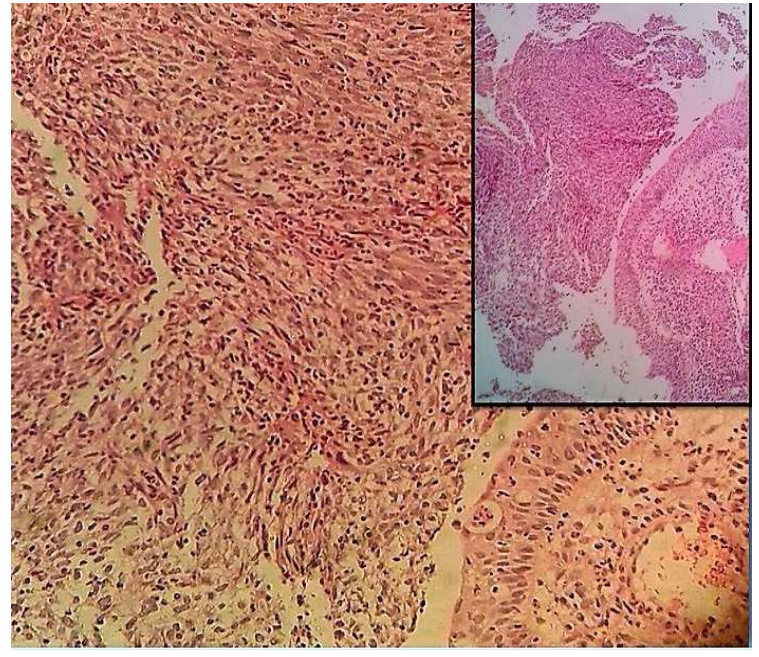

Figure-1: Tumour biopsy form nose showingmalignant spindle cell proliferation.

(Hematoxylin and Eosin, 20X and inset, 5X)

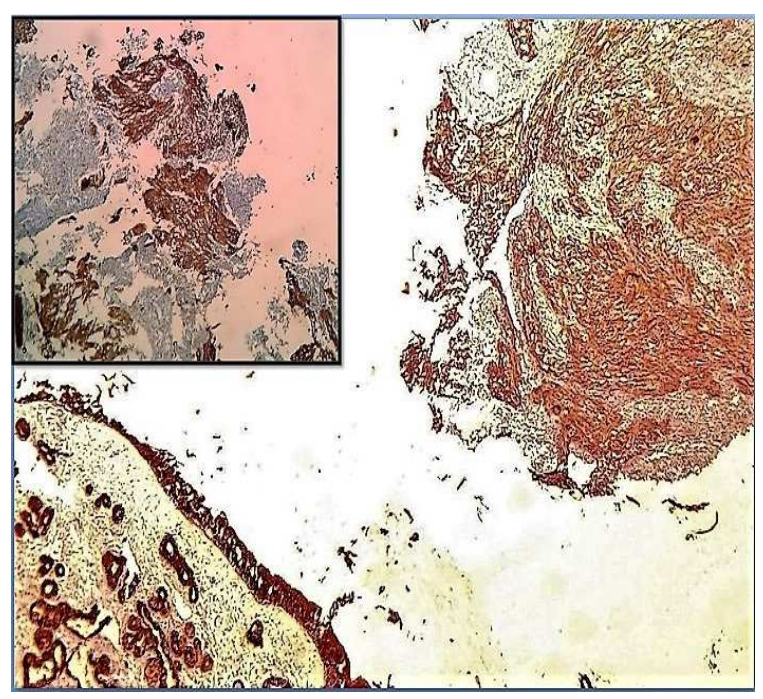

Figure-3: Tumour cells are pan-cytokeratin (CK) positive and (INSET) vimentin positive.

(Immunohistochemistry, 10X)

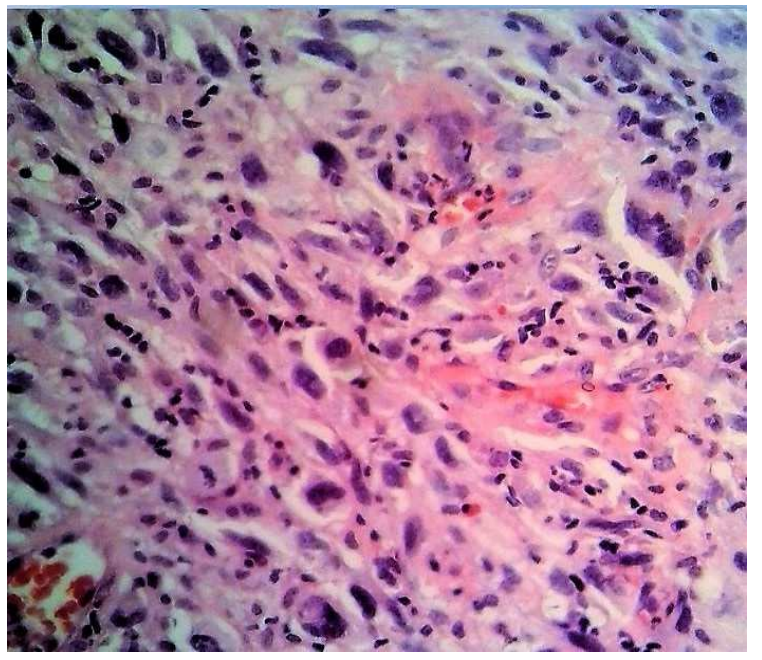

Figure-2: Spindle tumour cells showing hyperchromatic and irregular nuclei, prominent nucleoli and eosinophilic cytoplasm. (Hematoxylin and Eosin, 40X)

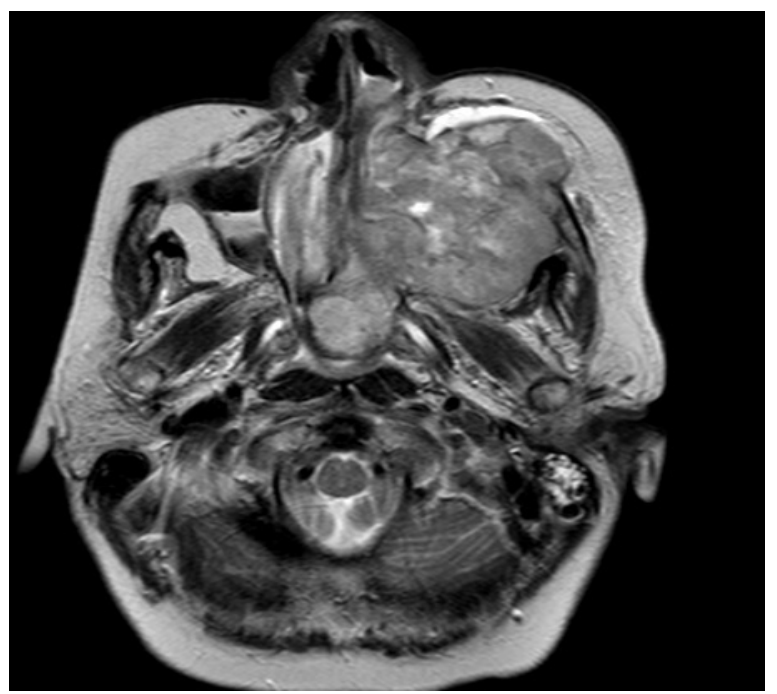

Figure-4: MRI revealing a large heterogenous mass in the maxillary sinus and with destruction of all its walls. 


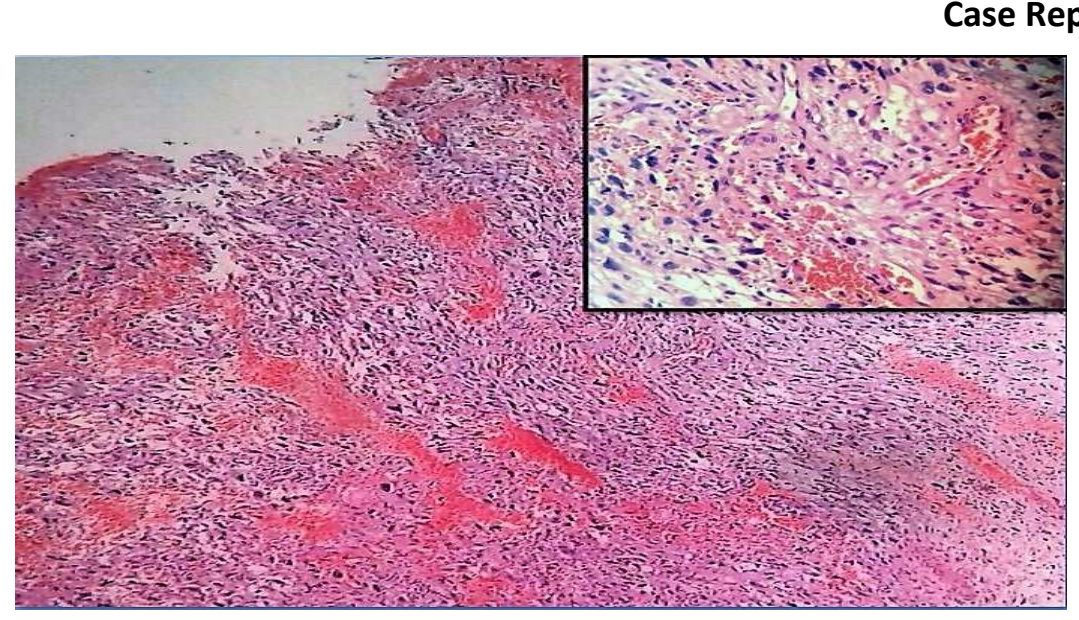

Figure-5: Malignant spindle cell proliferation in a hemorrhagic background. (Hematoxylin and Eosin, 10X) Spindle cells with hyperchromatic nuclei, blood vessels and neutrophils mimicking granulation tissue. (INSET, Hematoxylin and Eosin, 40X)

The patient was referred to higher centre for further management but expired before being posted for the surgery.

\section{Discussion}

SPCC is a unique biphasic variant of SCC that histologically exhibits spindled tumour cells. Many alternate terms like carcinosarcoma, pseudo sarcoma, pleomorphic carcinoma, and metaplastic carcinoma had been used for it. It is a rare biphasic tumour which can involveany organ including head and neck region. Many of the characteristic features of this tumour are similar to the conventional SCC $[1,2]$.

SPCC is more common in elderly people in $5^{\text {th }}$ to $6^{\text {th }}$ decades with male preponderance (Male: female-7:1). It also has a strong association with smoking and alcoholism $[1,2,3]$

In head and neck, larynx (glottis) is the most common primary site, followed by the oral cavity (tongue, floor of mouth, gingiva). Less common sites include the hypopharynx, oropharynx, sinuses and nasal cavity. They usually present as polypoidal, fleshy, exophytic masses with average size of $2 \mathrm{~cm}[1,2]$. Clinically they present as locally advanced stage, most tumours being $\mathrm{T} 3$ or T4 at presentation. It is an aggressive tumour if not treated in time [4].

Most significant are the histological features of this tumour, characterized by a biphasic pattern of SCC and generally muchlarger component of malignant spindled cells, reminiscent of a sarcoma. The squamous component may be scant oreven absent on light microscopy. The spindled cells may be bland and regular or may be markedly pleomorphic with multinucleated giant tumor cells with wide variety of architectural patterns including fascicular, storeiform, lacelike, or myxoid and rarely sarcomatous differentiation, such as osteosarcomatous, chrondrosarcomatous, or rhabdomyosar comatous may be seen. Mitotic activity can vary. The tumour can show abundant small vessels with plump endothelial cells and numerous inflammatory cells, particularly neutrophils and can closely mimic exuberant granulation tissue. This makes thediagnosis very difficult and requires immunohistochemical (IHC) and ultra structural studies.

Differential diagnosis of SPCC is therefore extremely exhaustive as many tumours of spindle cell morphology resemble it. Non neoplastic lesions like exuberant granulation tissue, nodular fasciitis, sinonasal polyp with stromal atypia, borderline tumours like inflammatory my ofibroblastic tumour and malignant tumours like spindle cell myoepithelioma or carcinoma, solitary fibrous tumor, Kaposi's sarcoma, synovial sarcoma, angiosarcoma, fibrosarcoma, leiomyosarcoma, malignant peripheral nerve sheath tumour (MPNST), undifferentiated pleomorphic sarcoma (malignant fibrous histiocytoma), spindle cell melanoma, spindle cell variant of rhabdomyosarcoma, high-grade transformation of dermatofibrosarcoma protuberance.

Non neoplastic and benign low grade tumours can be differentiated by clinical history, absence of atypical mitoses, cellularity, nuclear features and organization while malignant tumours often require further immunohisto chemistry with desmin, muscle specific actin, MYO-D, HMB 45, S100, calponin, CD 10, CD $31, \mathrm{CD} 34$, myogenin, CD 99 etc for confirmation $[1,5]$.

SPCC stain with both epithelial and mesenchymal markers. AE1/AE3 (pancytokeratin) is positive in anywhere from $26 \%$ to $62 \%$ of cases. Epithelial 
membrane antigen (EMA), p63, CK5/6 may also be positive and useful epithelial markers. Almost $100 \%$ of cases are positive for vimentin. Some may also express markers of muscle differentiation like SMA. SMA, Desmin, muscle specific actin can be positive in 1-30\% cases. One of our cases was also SMA positive [6,7].

Ultra structurally, the presence of cytokeratin type intermediate filaments, cadherins, desmosomes, tight junctions or adherent junctions also supports an epithelial origin of these tumors [8].

Tumours with positivity for both epithelial and mesenchymal markers such as inflammatory my fibroblastic tumours and other malignant biphasic tumours such as Synovial Sarcoma (SS), and epitheliod sarcoma need to be discussed here. The presence of surface epithelial dysplasia, squamoid differentiation within the tumour, tumour giant cells, collagenous stroma, a high degree of anaplasia and atypical mitoses are all features that should more strongly suggest a diagnosis of SPCC [1].

There is absence of invasive growth, markedly atypical cytologic features and atypical mitotic figures in inflammatory my fibroblastic tumours which are also rich in plasma cells and more strongly positive for SMA and muscle specific actin. Epithelial markers are more diffusely positive in SPCC than in SS which shows only focal CK or EMA positivity. Other markers such as CD 99, S100, Bcl2 are more likely to be positive in SS.Epitheliod sarcoma is also very rare shows sheets of polygonal cells with variable intervening stroma with stronger EMA, desmin, CD 34 positivity than SPCC. $[5,9]$

Two hypotheses for the origin of these biphasic tumours have been proposed. The convergence hypothesis suggests that they originate from 2 stem-cell lines, whereas the divergence hypothesis which is more acceptable by new research suggests that the tumor originates from a single totipotent stem cell that differentiates into epithelial and sarcomatous components [10].

Epithelial-mesenchymal transition theory suggests that morphologic and molecular change in epithelial cells occurs as a result of trans differentiation toward the mesenchymal type of cells and the malignant epithelial cells that differentiate into spindle cells lose their malignant phenotype, but they still retain the desmoplastic stroma that is essential for tumor proliferation and metastasis. Four theories have been proposed to explain the histogenetic concept of spindle cell carcinoma. The first theory suggested that the

\section{Case Report}

spindle cells and epithelial cells arose simultaneously from separate stem cells. The second theory explained that the nature of the spindle cell component was an atypical reactive proliferation of the stroma and the name, "pseudosarcoma" was proposed. The third theory explained that both the spindle and epithelial components had the same monoclonal origin.

The fourth theory explained that the spindle component was caused by the de-differentiation of the tumour cells. The dual antigen positivity suggests that the cells are in transition and it may represent sarcomatous metaplasia of a squamous cell carcinoma [11].

Carcinosarcomas are highly infiltrative and metastasizing and their behaviour is similar to that of poorly differentiated carcinomas. Interestingly metastases of carcinosarcoma usually contain squamous cells or a combination of squamous cells and spindle cells and they are rarely made up of spindle cells only [5].

No definite treatment protocol exists for this unique entity and tumour is primarily treated with aggressive, wide local excision. Adjuvant radiation and/or chemotherapy should be used in case of incomplete removal or positive surgical margins [12]. While other studies suggest that postoperative chemoradiation therapy improves patient prognosis and should be considered as the standard therapeutic modality [13].

\section{Conclusion}

Spindle cell carcinoma is a very rare and unique biphasic variant of squamous cell carcinoma. Diagnosis is challenging in head and neck because of different types of spindle cell lesions and requires immuno-histochemical and ultrastructural studies. Their behaviour is similar to that of poorly differentiated carcinomas. Tumour is primarily treated with aggressive, wide local excision, adjuvant radiation and/or chemotherapy used in case of incomplete removal or positive surgical margins.

\section{Funding: Nil, Conflict of interest: None initiated} Permission from IRB: Yes

\section{References}

1. Viswanathan S, Rahman K, Pallavi S, Sachin J, Patil A, Chaturvedi P, D'Cruz A, Agarwal J, Kane SV. Sarcomatoid (spindle cell) carcinoma of the head and neck mucosal region: a clinic pathologic review of 103 cases from a tertiary referral cancer centre. Head Neck Pathol. 2010 Dec; 4 (4): 265-75. doi: 10.1007/s12105010-0204-4. Epub 2010 Aug 22. 
2. Leventon GS, Evans HL. Sarcomatoid squamous cell carcinoma of the mucous membranes of the head and neck: a clinicopathologic study of 20 cases. Cancer. 1981 Aug 15;48(4):994-1003.

3. Thompson LD, Wieneke JA, Miettinen M, Heffner DK. Spindle cell (sarcomatoid) carcinomas of the larynx: a clinicopathologic study of 187 cases. Am J Surg Pathol. 2002 Feb;26(2):153-70.

4. Howard SN, Bond WR, Hong IS, Foss RD. Right maxillary sinus sarcomatoid carcinoma (sarcomatoid/ spindle cell carcinoma). Otolaryngol Head Neck Surg. 2007;137(2):355-57.doi:10.1016/j.otohns. 2007.03.002.

5. Lewis JS Jr. Spindle cell lesions--neoplastic or nonneoplastic?: spindle cell carcinoma and other atypical spindle cell lesions of the head and neck. Head Neck Pathol. 2008 Jun;2(2):103-10. doi: 10.1007/s12105008-0055-4. Epub 2008 May 28.

6. Lewis JE, Olsen KD, Sebo TJ.Spindle cell carcinoma of the larynx: review of 26 cases including DNA content and immunohistochemistry. Hum Pathol. 1997 Jun;28(6):664-73.

7. Ellis GL, Langloss JM, Heffner DK, Hyams VJ. Spindle-cell carcinoma of the aerodigestive tract. An immunohistochemical analysis of 21 cases. Am J Surg Pathol. 1987 May;11(5):335-42.

8. Choi HR, Sturgis EM, Rosenthal DI, Luna MA, Batsakis JG, El-Naggar AK.Sarcomatoid carcinoma of

\section{Case Report}

the head andneck: molecular evidence for the evolutionand progression from conventional squamouscell carcinoma. Am J Surg Pathol. 2003; 27 (9): 1216-20.

9. Fisher C. Immunohistochemistry in diagnosis of soft tissue tumours. Histopathology. 2011 Jun;58(7):100112. doi: 10.1111/j.1365-2559.2010.03707.x. Epub 2010 Dec 10.

10. Furuta Y, Nojima T, TerakuraN,Fukuda S, Inuyama $Y$. A rare case of carcinosarcoma of the maxillary sinus with osteosarcomatous differentiation. Auris Nasus Larynx. 2001; 28(S): 127-29.

11. Gupta R, Singh S, Hedau S, Nigam S, Das BC, Singh I, Mandal AK. Spindle cell carcinoma of head and neck: an immunohistochemical and molecular approach to its pathogenesis. J Clin Pathol. 2007 May; 60 (5):472-5. Epub 2006 May 26.

12. Iqbal MS, Paleri V, Brown J, Greystoke A, Dobrowsky W Kelly C, Kovarik J. Spindle cell carcinoma of the head and neck region: treatment and outcomes of 15 patients. Ecancer medical science. 2015 Nov 18;9:594. doi: 10.3332/ ecancer. 2015. 594. e Collection 2015.

13. Alem HB, AlNoury MK. Management of spindle cell carcinoma of the maxillary sinus: a case report and literature review. Am J Case Rep. 2014 Oct 24;15:4548. doi: 10.12659/AJCR.891007.

\section{How to cite this article?}

Mirza N, Haider N, Fatima S.Two cases of spindle cell carcinoma with a histopathological review of biphasic tumours of head and neck. Trop J Path Micro 2018; 4(4):344-348.doi:10. 17511/ jopm. 2018.i4.08 